\title{
Application of Photoshop on the Post-Processing of Building Works
}

\author{
Huang keqin ${ }^{1,}$, \\ ${ }^{1}$ Chongqinq College of Electronic Engineering, Faculty of Art Media,Chongqing,401331,China \\ ahkqin@126.com
}

\section{Keywords: Photoshop, Post-Processing, Building Works}

\begin{abstract}
Nowadays, graphic image editing and processing has been very common in people's life and work, such as building plans produced and people's digital photo processing. The most popular image processing software Photoshop, its function is very powerful, graphic design, color printing, graphic creation, web design, image processing, multimedia and video effects, and other fields are widely used. Architectural post-processing is part of the post-processing software application and as architectural renderings production staff, but not the use of Photoshop landscaping, repair, adjustment of three-dimensional or two-dimensional renderings is unthinkable. Thus, combined with years of teaching experience in architectural renderings of post-processing, image processing software for the role of architectural renderings in post-treatment and the use of some of the considerations in this are discussed.
\end{abstract}

\section{Introduction}

Adobe Photoshop is the company launched professional image processing software, to its powerful image processing, editing and good color performance, the popular image processing and graphic designers. Its simple operation and easy to learn, true and perfect material processing, color rich and colorful, and more importantly, it's very powerful, it was quickly used in building design, planning and design industry. In the field of architectural design, Photoshop superiority in architectural renderings late manifestations of energy is well known. However, as an architect, it also can be incorporated into its own program to the creation, rather than just as a means of program performance only.

Purely from the perspective of Photoshop, 3ds max graphic work for Photoshop provides a crude embryo for modification. Three-dimensional software like 3dsmax model has a strong production capacity, can fully reflect the three-dimensional structures and texture, but in the post-processing ambience and production aspects of the real background appeared to be inadequate, and if you use Photoshop to handle, just the background image of the final scene with 3dsmax output image can be fused, such as the use of people, cars, clouds, sky, trees and other field shooting pictures or pictures with synthetic environment, so that works ultimately has a natural, harmonious atmosphere, so than the traditional hand-drawn expression of specific, much more real, to facilitate simulation of the effect of a variety of lighting conditions, such as night lighting effects and noon sunlight, converting just two different colors, adjust the illumination can be achieved, also facilitate various modifications, partial unreasonable be easy to solve.

\section{Post-Processing Step}

Before use photoshop to make post-processing, we must first identify the problems rendering, only pinpoint problems, it can be a good solution to the problem. For example, an unsuccessful rendering, it exists in the following aspects. The whole image is too gray tone, the bright places do not light up the dark places do not go dark, not well reflect the brightness changes. Scenario modeling texture does not reflect well, such as curtains, sofas and floors modeling material. Down light lighting is too dark and making have no ceiling lights with the effect seemed more monotonous. Missing people, plants and other background, so that the whole image looks dull lifeless.

Conduct renderings post-processing effects start to start big, a good grasp of the overall relationship, this adjustment when local effect (for example, when adjusting materials and lights 
will not affect large effect) while the partial renderings adjustment is also easy to grasp.

Materials and lighting affect each other. The main material used to reflect light texture \# modeling scene is not only used to illuminate the scene, more well express a scene atmosphere. 3DMAX rendering output from the image, material and lighting always there unpleasantness place, which need to be adjusted later in the process.

Interior Design's background includes plants, humans and some other decorations. When selecting material generally choose sleek, color images can be coordinated with the main colors of the material, and then the screen needed to be adjusted in detail.

\section{Post-Processing Effects of Photoshop}

As can be seen from the outdoor architectural renderings production process, post processing photoshop has a very important role in the architectural renderings of production. The work of three-dimensional software just take photoshop for simple modification crude embryo, only after photoshop treatment, to get a real realistic scenes, therefore, it is no less preliminary modeling work. Since the latter part of the deal is final renderings production process a step, so its success is directly related to the overall success of renderings, which requires the operator to have a profound knowledge of art, can grasp the soul of the whole work. Photoshop summary procedure and specific applications in architectural renderings of post-processing can be broadly grouped into the following aspects.

When the complexity of the scene, a number of light when renderings obtained there will be some small defects or errors, if we return 3dmax readjust, time-consuming and laborious. At this time you can play photoshop expertise, using the repair tool and color adjustment tools to easily modify the model or defects caused by light settings generated. This is the first step working renderings post-processing.

Adjust the color and tone graphics, mainly refers to the use of photoshop brightness, contrast, hue, saturation, gradation, color balance, curves, color, tint adjustment commands to the graphics to be adjusted to give a clearer, more coherent tone color graphics this is the second step renderings post-processing.

As mentioned above, the image 3dmax render output, often just a simple crude embryo renderings, scenes monotonous, stiff, and the lack of level change, only its accession to the sky, trees, people, cars and other background, the entire rendering it look lively and interesting, full of vitality, of course, the work is also done by photoshop, this is the third step of post-processing work renderings. For example, the production of Halo, with light and rendering water droplets, fountain, rendering the rain scene, snow, hand-drawn look, so as to meet the needs of some special effects.

\section{The Role of Photoshop on Post-Processing and Processing Method}

Three-dimensional renderings of pre-production and post-production division process in two stages. The so-called pre-production generally refers to the use 3DMax and other three-dimensional software for modeling, editing materials, lighting, and, ultimately, to make a good showing effects rendering. 3D renderings but often there are some problems, such as color cast, light error, add background inconvenient, not even background, level is not clear, the sense of space is not strong, then you need to post-process 3D rendering .

Photographs, prints, etc., or the reality of the scene input through the data the camera to a computer there are often color deviation, 3D renderings have the same problem, so, 3D rendering post-treatment should first be on rendering the overall tone of light and shade, color tends to be determined and thus set the tone for a partial correction and add background. At the same time, overall adjustment will also help strengthen the sense of depth and sense of space images. Color correction renderings are usually two circumstances: a building should reflect the true color of indoor and outdoor environment and appropriate landscaping, allowing users to feel comfortable environment, such as the sky to blue, to green grass, colorful flowers to it. When you can use color correction commands to adjust the hue and color saturation; b made into a special color effects, 
such as the interior color with special lighting or large areas of a certain color background, which will impact on the indoor environment or goods in. renderings must pay attention to post-processing. But no matter what kind of situation, you need to apply the color adjustment commands; as corrected overall image color cast, you can use the curve command; adjust hue, saturation, color, etc., you can use the Hue Saturation command; local color adjustment, You can use Select color or replace color command.

In 3DMax although you can also add some background, but it is not easy, and sometimes there are some problems after output rendering, so use Photoshop to add background is an important task for post-processing. If Interior Design, generally need to add background bonsai, vases, ornaments, etc., if it is an outdoor renderings, background and more complicated, such as the sky, trees, grass, pedestrians, vehicles, surrounding buildings, even lakes and other water. Sometimes they do not have the required background material readily available, but also for production, such as some artifacts, books or the like fountains.

In rendering process, the most difficult is probably the number of renderings of light or shadow processing and produced. For example, Interior Design, renderings, if there can be lighting locations, such as windows, balconies, day or night, so be sure to be reflected. If it is daytime, outdoor scene is bright and clear, and the impact on indoor items. If it is night, and bright indoor light, the window will generate an image of an indoor scene, while light indoor room light items only, then need to be in accordance with the type of lamp lights glow production. For exterior renderings, the light source than a single, only sunlight, so outdoor background are projected position of the sun as a reference. If the outdoor background in the lake, the water pools, should consider the surrounding scenery reflection in the water.

After the output 3D renderings, images often have some problems, such as shadow and light direction background and background does not match the wall surface seams obvious lack of texture and other materials, then the need to use Photoshop for trimming. During the trimmed-image, generally using the stamp tool, but for large areas of color, the same pattern area, you can use the selection tool to copy (this time should use the Alt key copy, rather than the application Ctrl + C to copy, otherwise it will Add layer). If the problem is a light texture or material problems, usually you need to select the existing content, and then use the Pho-toshop tools and filters to re-create.

Dimensional renderings general layout for the performance of the building, so in the production of three-dimensional renderings relative terms, more emphasis on the function of the building rather than the actual results show, in the post-production is relatively simple. Post-production two-dimensional renderings generally include two aspects: Material production and filling. As the size chart pattern in the ground, the wall elevation pattern making and filling, if readily available material can be used directly. In the size chart, with respect to the different functions of different rooms need to lay flooring, such as marble flooring shop bedroom, living room, kitchen and bathroom tiles, etc., then you can make your own common wood floors, ceramic tile, marble and other ground can use Photoshop for production. Production and add background. Two-dimensional renderings of the plan refers to the general background of the furniture, kitchen utensils, toilet facilities, etc., often add elevation surroundings of buildings such as the sky, trees, pedestrians, vehicles, etc., but with different three-dimensional renderings, elevations of these background no sense of space.

\section{Conclusion}

With the rapid development of the construction industry, the performance of the construction industry has matured and the division of labor has become increasingly refined. Some professional renderings produced renderings companies have divided into pre-modeling, rendering and post-processing step 3. Pre-built the main mode is to use the software to create the building model 3dmax and given material, arranged the lighting, then render as a bitmap software. Since photoshop software rendered image is not perfect, need to make up for some deficiencies through post-processing and production environment with King, in real simulation of real space or 
environment, this process is the post-processing work, usually needs to be done in photoshop. Post-processing determines the final effect chart performance results and success artistic level.

\section{References}

[1] Guo Jianghui. Computer-aided design applications in civil engineering [J]. Shanxi Construction, 2006, 32 (16): 52-53.

[2] Li Shaoyong. 3ds max 6 upholstery skills Gifted [M]. Beijing: Beijing Hope Electronic Press, 2004.302-306.

[3] Zhang Pan, Tang Yuanlin, Hong Qing, Zhu Xiaoping. Experimental physics innovation consciousness and ability of [J]. Physical Experiment 2001 (04)

[4] Liu Zhizhong. Governance oil pollution caused by the basic ways [J]. Environmental Science. 1990 (04)

[5] Wen Guangjun. Holography test piezoelectric crystal $\sigma-\mathrm{V}$ curve [J]. Laser Medicine. 1991 (05) 